\title{
Medidas dos Níveis de Pressão do Balonete em Unidade de Terapia Intensiva: Considerações sobre os Benefícios do Treinamento*
}

\author{
Measurement of Tube Cuff Pressure Levels in Intensive \\ Care Unit: Considerations on the Benefits of Training
}

\author{
Silvia Renata Rezek Juliano', Maria Cecilia Rezek Juliano², Jose Paulo Cividanes³, \\ João Geraldo Simões Houly ${ }^{4}$, Otavio Celso Eluf Gebara ${ }^{5}$, Gil Vicente L. Cividanes ${ }^{6}$, Elaine C. Catão ${ }^{7}$
}

\section{RESUMO}

JUSTIFICATIVA E OBJETIVOS: A pressão do balonete transmitida diretamente na parede da traquéia de forma irregular pode ocasionar lesões e levar a broncoaspiração. O objetivo deste estudo foi demonstrar que ao implantar uma rotina de mensuração da pressão do balonete, obtém-se controle fidedigno para manter as medidas dentro dos parâmetros considerados seguros, evitando assim, as complicações descritas.

MÉTODO: Foram avaliadas 3195 medidas de pressão de balonete em 1194 pacientes dos sexos masculino e

1. Fisioterapeuta Coordenadora do Serviço de Fisioterapia do Hospital Santa Paula e Especializada em Fisioterapia Cardiorrespiratória pelo InCor HC-FMUSP.

2. Fisioterapeuta Coordenadora do Serviço de Fisioterapia do Hospital Santa Paula.

3. Médico-Gerente de Qualidade do Hospital Santa Paula

4. Médico Coordenador da Unidade de Terapia Intensiva Adulto do Hospital Santa Paula.

5. Médico Coordenador da Unidade Coronariana do Hospital Santa Paula.

6. Médico Coordenador da Unidade de Terapia Intensiva B do Hospital Santa Paula.

7. Psicóloga, Doutora em Psicologia Escolar e Desenvolvimento Humano, Docente da UNISÃOPAULO, Coordenadora da ALAMOCBrasil

${ }^{*}$ Recebido da Unidade de Terapia Intensiva do Hospital Santa Paula, São Paulo, SP

Apresentado em 01 de junho de 2007

Aceito para publicação em 20 de agosto de 2007

Endereço para correspondência:

Serviço de Fisioterapia - Hospital Santa Paula

Avenida: Santo Amaro $n^{\circ} 2468$ - $3^{\circ}$ Andar Ala B

04556-100 - São Paulo, SP

Fone: (11) 3040-8166

E-mail: fisioterapia@santapaula.com.br

(C)Associação de Medicina Intensiva Brasileira, 2007 feminino, internados nas unidades de terapia intensiva (UTI) e coronariana (UC), que estavam sob ventilação mecânica com uso de prótese endotraqueal e cânula de traqueostomia, nos períodos matutino e vespertino.

RESULTADOS: Durante o período de março a agosto de 2005 foi realizado acompanhamento das medidas colhidas pelos profissionais de fisioterapia e observouse que as medidas foram irregulares, em média, em $80 \%$ dos casos. Diante desse fato foi elaborado um programa de treinamento, com foco nas Equipes de Enfermagem das UTI e UC, que consistiu na orientação dos procedimentos adequados realizados à beira do leito (treinamento em loco). Os treinamentos foram realizados em dois períodos (matutino e vespertino) para abranger toda a equipe.

CONCLUSÕES: Sugere-se a necessidade da vigilância das pressões do balonete através da implantação de uma rotina de mensurações matutina, vespertina e noturna, como meio profilático, para prevenir as possíveis complicações da pressão de balão da prótese traqueal.

Unitermos: balão endotraqueal, balonete traqueal, intubação, traqueostomia

\section{SUMMARY}

BACKGROUND AND OBJECTIVES: The tube cuff pressure directly transmitted on the tracheal wall in an irregular form can cause injuries and lead to bronchoaspiration. The aim of this study was to demonstrate that the implementation of routine tube cuff pressure measurements result in a reliable control to maintain the measurements within the parameters considered safe, thus preventing the described complications.

METHODS: A total of 3,195 tube cuff measurements 
were obtained from 1,194 male and female patients admitted at the Intensive Care Unit (ICU) and Coronary Unit (CU), who were undergoing mechanical ventilation with endotracheal prosthesis and tracheotomy cannula, during the morning and afternoon periods.

RESULTS: From March to August 2005 the follow-up of the measurements obtained by the physical therapy professionals was carried out and it was observed that the measurements were irregular, on average, in $80 \%$ of the cases. Thus, a training program was established, which was focused on the Nursing Teams of the ICU and $\mathrm{CU}$, consisting in providing directions for the adequate procedures performed at the bedside (in loco training). The training procedures were carried out at two different periods (morning and afternoon) in order to include the whole team.

CONCLUSIONS: It is suggested that it is necessary to monitor tube cuff pressure through the implementation of routine measurements in the morning, afternoon and evening periods as a prophylactic measure, in order to prevent the possible complications of tracheal prosthesis balloon pressure.

Key Words: Endotracheal balloon, intubation, tracheotomy, tracheal tube.

\section{INTRODUÇÃO}

Sabe-se que nas unidades de terapia intensiva (UTI) é comum encontrar pacientes que necessitam de suporte ventilatório mecânico invasivo através de prótese traqueal ${ }^{1,2}$.

As próteses traqueais mais utilizadas são os tubos endotraqueais e as cânulas de traqueostomia².

Para uma ventilação mecânica adequada, sem que haja escape aéreo, as próteses endotraqueais e de traqueostomia possuem na sua porção distal um balonete, cuja função é selar a via aérea ${ }^{3,4}$.

Para que o paciente submetido a este tipo de ventilação mecânica não sofra complicações da mucosa traqueal ou broncoaspiração torna-se necessário observar algumas variáveis como, por exemplo, o tempo de intubação e a pressão na parede lateral da traquéia ${ }^{5-7}$. A pressão de perfusão sanguínea da mucosa traqueal situa-se entre 25 e $35 \mathrm{mmHg}^{5,8}$. Quando é feita a medida em $\mathrm{cmH}_{2} \mathrm{O}$, esses valores não devem ultrapassar 20 e $30 \mathrm{cmH}_{2} \mathrm{O}^{9}$. Pressões superiores a $30 \mathrm{cmH}_{2} \mathrm{O}$ podem gerar lesões na parede da traquéia e pressões menores que $20 \mathrm{cmH}_{2} \mathrm{O}$ podem levar a broncoaspiração ${ }^{8-10}$. Observa-se, atualmente, que os estudos demonstram a importância de se manter o controle da pressão pró- ximo a normalidade para evitar as possíveis complicações do balão da prótese endotraquea| ${ }^{6,11}$.

Não é habitual encontrar na rotina hospitalar mensuração da pressão de balonete. A literatura específica da área não apresenta um total padrão de medidas que devam ser observadas durante o período de intubação do paciente e estudos tem demonstrado que a palpação digital do balonete externo é insuficiente para detectar alterações da pressão ${ }^{1,3,12}$. Contudo, no Segundo Consenso de Ventilação Mecânica está estabelecido que as medidas devem ser verificadas a cada 12 horas $^{9}$.

Neste contexto, e tendo como base a prática diária da fisioterapia hospitalar, o objetivo do presente estudo foi demonstrar que ao implantar uma rotina de mensuração da pressão de balonete, obtém-se controle fidedigno para manter as medidas dentro dos parâmetros considerados seguros, evitando complicações.

Nesta instituição as medidas da pressão de balonete são realizadas diariamente, no período matutino, vespertino e noturno, com uso de um cuffômetro. As suas medidas verificadas com freqüência demonstraram que existe variação da pressão $<20 \mathrm{cmH}_{2} \mathrm{O}$ e $>30 \mathrm{cmH}_{2} \mathrm{O}$.

\section{MÉTODO}

Após aprovação do Comitê de Ética foi realizado estudo descritivo, prospectivo, no período março de 2005 à dezembro de 2005, nas unidades de terapia intensiva (UTI) e coronariana (UC) do Hospital Santa Paula.

Foram avaliadas 3195 medidas de pressão de balonete em 1194 pacientes dos sexos masculino e feminino, internados nas unidades e que estavam sob ventilação mecânica com uso de prótese endotraqueal e cânula de traqueostomia, nos períodos matutino e vespertino.

A pressão do balonete foi mensurada utilizando um aparelho manual (cuffômetro), com graduação de 0 a $120 \mathrm{cmH}_{2} \mathrm{O}$, mantendo as normativas de calibração \pm $0,49 \%$, que conectado ao balonete permitiu a sua insuflação e desinsuflação. Os valores de referência foram mantidos entre 20 e $30 \mathrm{cmH}_{2} \mathrm{O}$.

O fisioterapeuta ajustava as pressões quando essas estavam fora dos valores considerados normais, não permitindo o vazamento de ar. Nos casos em que a cânula apresentava escape durante a ventilação mecânica, ajustava-se a pressão até não haver mais escape. Foram consideradas também medidas irregulares (medidas acima ou abaixo dos parâmetros de referência) e regulares (medidas dentro dos parâmetros de referencia). A cânula traqueal utilizada foi da marca Rush, preferencialmente números 7,5 a 8,5 e a cânula de traqueosto- 
mia da marca Portex, preferencialmente números 7,5 a 8,5 com balão de baixa pressão.

\section{RESULTADOS}

Durante os primeiros seis meses (março de 2005 a agosto de 2005) foi realizado o acompanhamento das medidas colhidas pelos profissionais de fisioterapia e observou-se que as medidas irregulares foram observadas, em média, em $80 \%$ dos casos (Figura 1).

Diante do exposto foi elaborado um programa de treinamento com foco nas Equipes de Enfermagem das unidades de terapia intensiva e coronariana que consistiu na orientação dos procedimentos adequados rea-

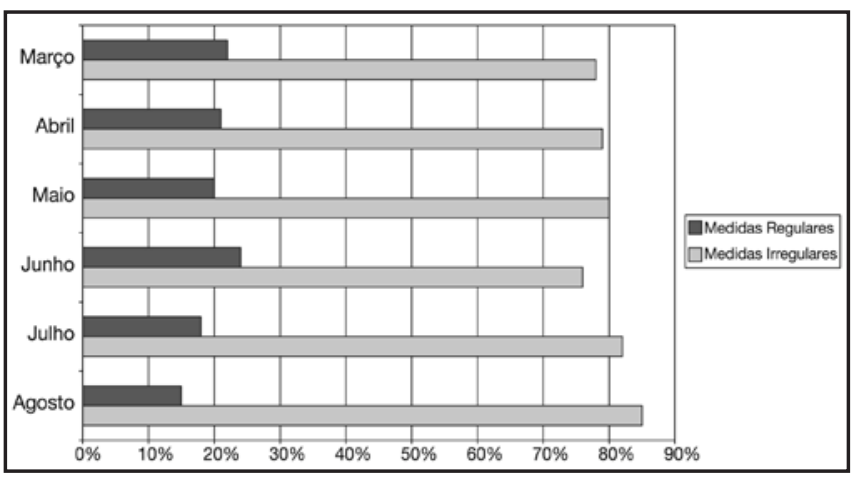

Figura 1 - Distribuição em Percentual das Medidas Regulares e Irregulares Antes do Treinamento. lizada à beira do leito (treinamento em loco). Tais treinamentos foram realizados em dois períodos (matutino e vespertino) para abranger toda a equipe (Figura 2). Ao final do primeiro mês de treinamento (setembro/2005) observou-se diminuição de $20 \%$ das irregularidades. O cálculo do teste Qui-quadrado de independência, realizado com as medidas de pressão do balonete regulares e irregulares de agosto de 2005 (antes do treinamento) e setembro de 2005 (após o treinamento) foi de 56,32 para um grau de liberdade e significância de 0,00\% (nível significativo de 0,05$)$. Portanto, pode-se observar que o treinamento realizado contribuiu para a melhoria do controle dos níveis de pressão do balonete (Figura 3).

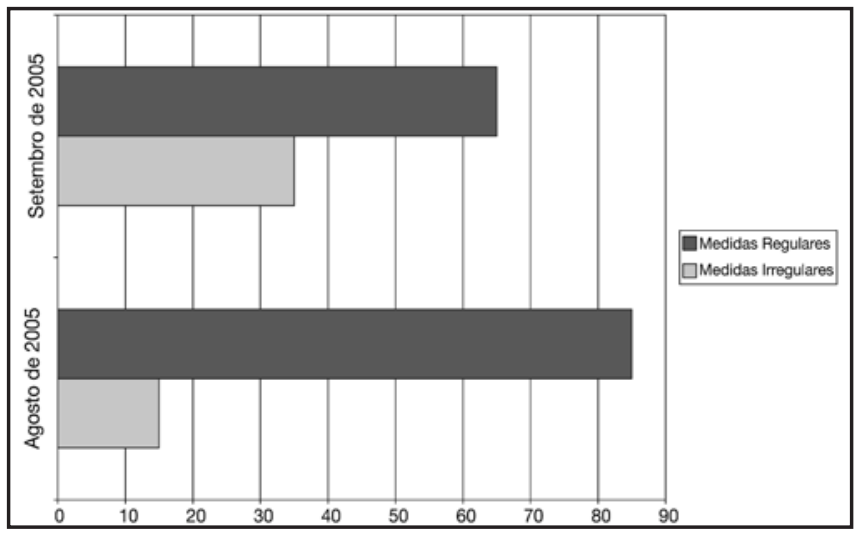

Figura 3 - Comparação Percentual de Medidas Regulares e Irregulares Antes e Após o Treinamento.

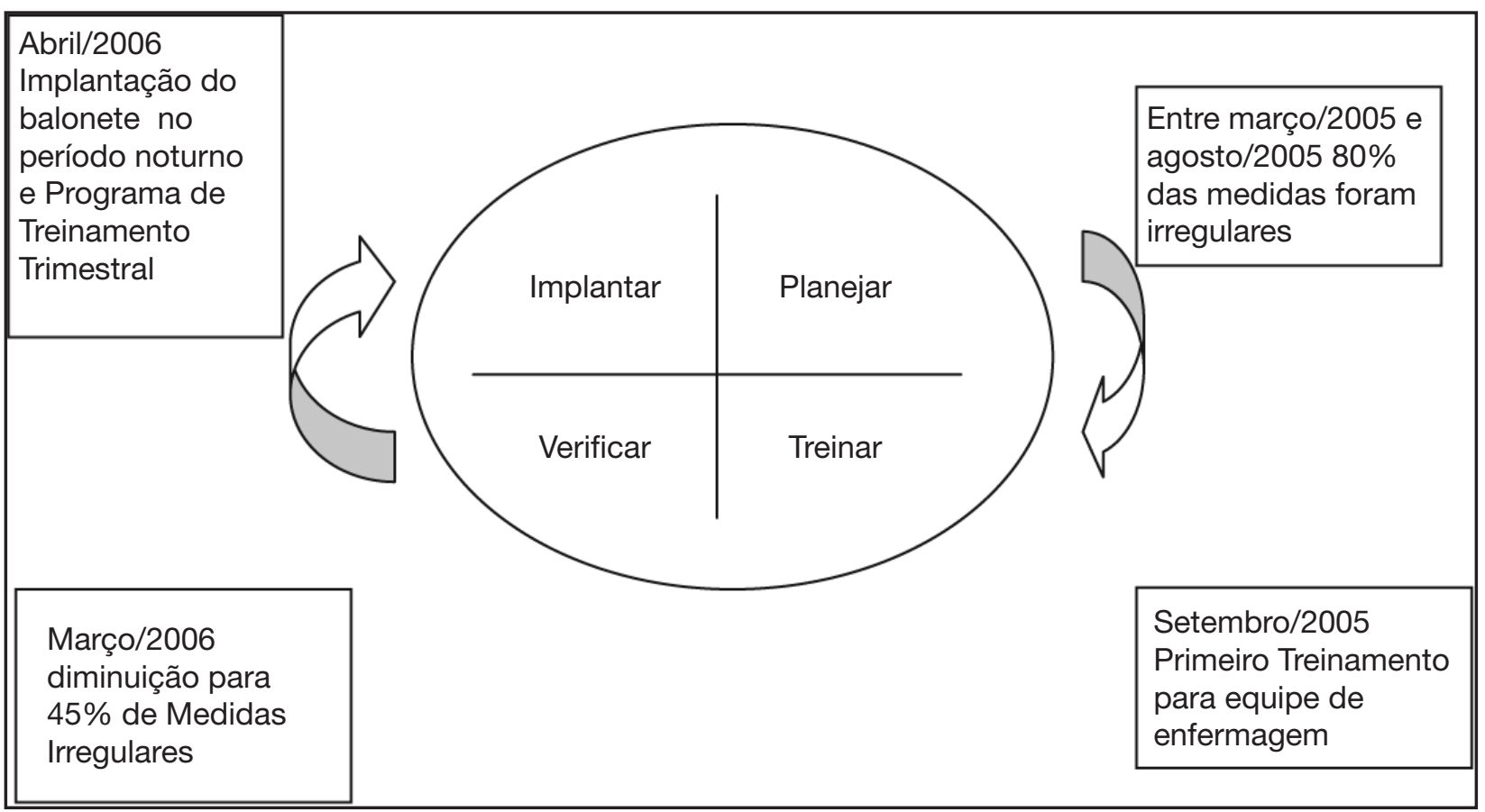

Figura 2 - Implantação da Rotina da Medida do Balonete no Período do Estudo. 
Foi observado nos dados colhidos no período do estudo (Figura 4) que existiam irregularidades tanto acima e quanto abaixo dos parâmetros de segurança ( $>20 \mathrm{e}<30 \mathrm{cmH}_{2} \mathrm{O}$ ), passou-se então, a discriminar, em todas as mensurações, níveis acima e abaixo dos limites aceitáveis.

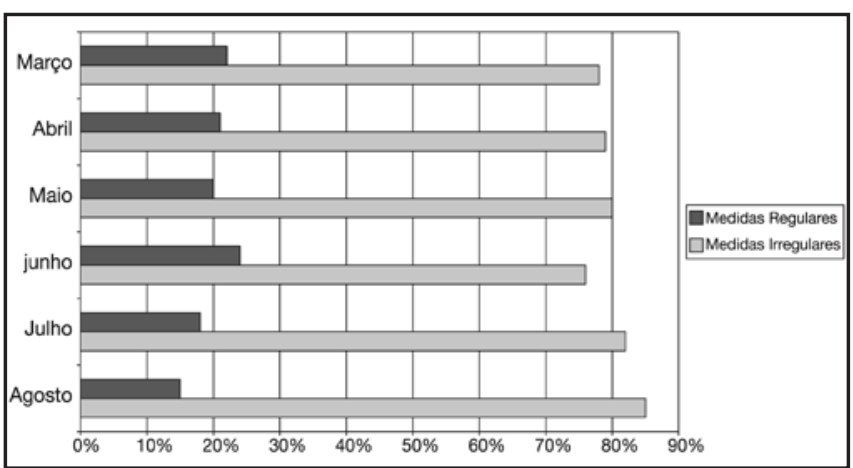

Figura 4 - Distribuição em Percentual das Medidas Regulares e Irregulares - Após o Treinamento.

Outro dado que chamou atenção foi a diferença estatisticamente significativa encontrada entre as mensurações realizadas nos períodos matutino e vespertino (Qui-quadrado de independência igual a 36,80 para um grau de liberdade e significância de 0,00\% - significativo ao nível de 0,05$)$, entre os meses de setembro a dezembro de 2005 (Figura 5). A partir deste dado foi instituída a mensuração da pressão do balonete também no período noturno. Tal procedimento contraria um estudo realizado por Eid ${ }^{1}$ com 102 pacientes, nos quais foram coletadas 612 medidas de pressão do balonete (períodos matutino, vespertino e noturno), sendo observadas diferenças estatisticamente significativas apenas para os períodos matutino e vespertino, fato que levou a exclusão da medida no período noturno.

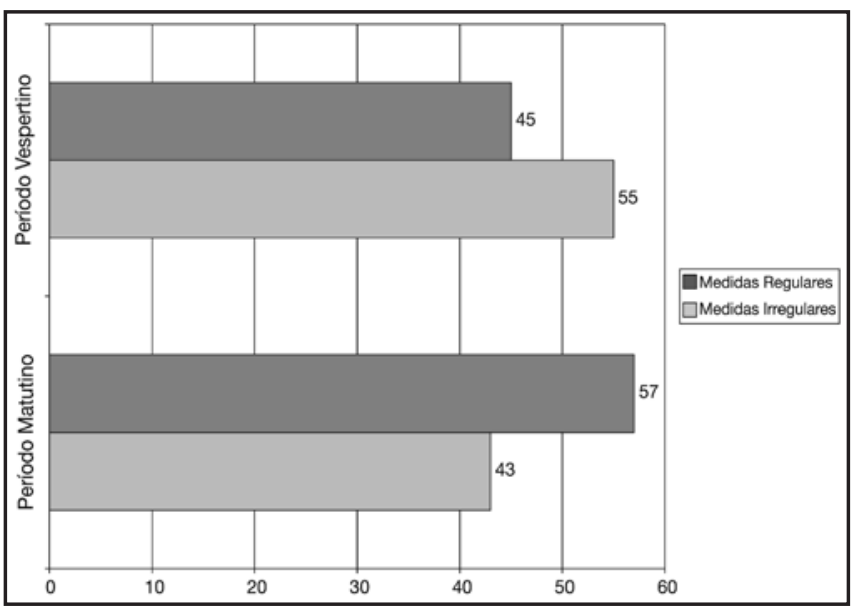

Figura 5 - Comparação em Percentual entre Medidas Regulares e Irregulares, nos Períodos Matutino e Vespertino.
Diante do exposto, foi instituída a prática de mensuração da pressão do balonete nos períodos matutino, vespertino e noturno e a realização do Programa de Treinamento Trimestral.

\section{CONCLUSÃO}

A necessidade de intubação traqueal por mais de 24 horas, pode apresentar certo grau de sofrimento da mucosa laringotraqueal ${ }^{5,10,13,14}$. Existem estudos evidenciando alterações na mucosa traqueal após 2 horas de intubação, caracterizando lesões precoces, sendo o balonete um fator preditor dessas complicações ${ }^{10}$. Vários fatores influenciam a pressão do balonete, entre o tipo e o tamanho do tubo traqueal ${ }^{6,11}$.

São necessários controles freqüentes e manutenção da pressão do balonete dentro da variação de valores aceitáveis. Pressões maiores que $25 \mathrm{cmH}_{2} \mathrm{O}$ evita com segurança a aspiração do conteúdo orofaríngeo e gastroesofágico, considerando-se $20 \mathrm{cmH}_{2} \mathrm{O}$ como limite mínimo, abaixo do qual poderia ocorrer broncoaspiração e pressão maior que $30 \mathrm{cmH}_{2} \mathrm{O}$ já comprometeria a perfusão da traquéia ${ }^{2,8,12,15}$. Recomenda-se que ao insuflar o balonete, deve-se usar menor quantidade de ar capaz de vedar o espaço entre o balonete e a parede traqueal ${ }^{12}$.

Não são rotineiras as mensurações destas pressões nas UTI, possivelmente pelo desconhecimento ou despreocupação dos profissionais de terapia intensiva com esta questão.

Os resultados encontrados neste estudo demonstraram as diferenças estatísticas de medidas irregulares mensuradas nos períodos matutino e vespertino.

Sugere-se que é necessária a vigilância das pressões do balonete através da implantação de uma rotina de mensurações matutina, vespertina e noturna, como medida profilática, para prevenir as possíveis complicações da pressão de balão da prótese traqueal.

\section{REFERÊNCIAS}

01. Eid R, Medeiros S, Kanda S et al - Mensuração da Pressão de Cuff em Terapia Intensiva. Anais do X Congresso Paulista de Pneumologia e Tisiologia, 2003.

02. Cordeiro AM, Shin SH, Fernandes Ide C et al - Incidence and endoscopic characteristics of airway injuries associated endotracheal intubation in children. Rev Assoc Med Bras, 2004;54:87-92.

03. Mendes FF, Hintz L, Bredemeier Neto F - Volumes e pressão do balonete do tubo traqueal para oclusão da traquéia. Rev Bras Anestesiol. 1996;46:103-106

04. Norris CM - Laryngeal and tracheal trauma in association with intubation JFORL J Fr Otorhinolaryngol Audiophonol Chir Maxillofac, 1974;23:163-167.

05. Messahel BF - Total tracheal obliteration after intubation with a lowpressure cuffed tracheal tube. Br J Anaesth, 1994;73:697-699. 
06. Stanley TH, Liu WS - Tracheostomy and endotracheal tube cuff volume and pressure changes during thoracic operations. Ann Thorac Surg, 1975;20:144-151.

07. Pattnaik SK, Bodra R - Ballotability of cuff to confirm the correct intratracheal position of the endotracheal tube in the intensive care. Eur $J$ Anaesthesiol, 2000;17:587-590.

08. Barbosa PMK, Santos BMO - Determinação do volume de ar no "cuff" de sondas endotraqueais. R Bras Enferm, 1996;49:225-238

09. II Consenso Brasileiro de Ventilação Mecânica. J Pneumologia, 2000;26: S1-S68.

10. Kaneko M - Fisioterapia na Ventilação Mecânica Convencional, em: Knobel E - Condutas no Paciente Grave: São Paulo: Editora Atheneu, 1998;1599-1609.
11. Passos E, Castilho VG - Papel da enfermagem na assistência ao paciente em ventilação mecânica. J Pneumol, 2000;26:27-34

12. Navarro LHC, Braz JRC, Pletsch AK et al - Estudo comparativo das pressões dos balonetes de tubos traqueais contendo ou não válvula reguladora de pressão de Lanz. Rev Bras Anestesiol, 2001;51:17-27.

13. Medalha S, Oliveira LC, Godoy I - Avaliação da pressão no balonete das cânulas endotraqueais e de traqueostomia em pacientes na Unidade de Terapia Intensiva. RBTI, 1999;11:90-93.

14. Yang $\mathrm{KL}$ - Tracheal stenosis after a brief intubation. Anesth Analg, 1995;80:625-627.

15. Braz JR, Navarro LH, Takata IH et al - Endotracheal tube cuff pressure: need for precise measurement. Sao Paulo Med J, 1999;117:243-247. 